\title{
THE FRATTINI ARGUMENT AND $t$-GROUPS
}

\author{
BEN BREWSTER AND SURINDER SEHGAL
}

\begin{abstract}
If enough subgroups of a group satisfy the Frattini argument in the group, then normality is a transitive relation within the group. Subgroup functors are used to specify what enough is.
\end{abstract}

1. Introduction. This article is an outgrowth of the investigation into functors which satisfy the Frattini argument. By a functor, we mean an association to each group $G$, a collection $f(G)$ of subgroups of $G$ such that if $\alpha: G \rightarrow \bar{G}$ is a monomorphism, then $f\left(G^{\alpha}\right)=\left\{U^{\alpha} \mid U \in f(G)\right\}$. Several types of functors have been explicitly formulated in the literature, e.g. Gaschütz functors in [1] and Sylow functions in [10]. Of course, the idea has been implicit when associating to a group, its Sylow subgroups, its system normalizers or its $\mathcal{F}$-injectors, where $\mathcal{F}$ is a Fitting class.

In attempting to better understand the nature of injectors for Fitting classes, the notion of a Fitting functor evolved in [2]. A Fitting functor is a functor $f$ which satisfies the additional property: if $N \triangleleft G$, then $f(N)=\{U \cap N \mid U \in f(G)\}$.

If $f$ is a Fitting functor and $G$ is a group, then $f$ satisfies the Frattini argument in $G$ provided for each $U \in f(G)$ and each $K \triangleleft G, G=K \cdot N_{G}(U \cap K)$. Theorem 7.2 of [2] and Theorem 3.10 of [3] give interesting characterizations of Fitting functors which satisfy the Frattini argument in each finite solvable group. Noting the general nature of the proofs of these results, it seemed of interest to investigate the groups in which a given functor satisfies the Frattini argument. This is the context in which the groups in which normality is a transitive relation appeared. In $\S 2$ we forge this connection and in $\S 3$ we investigate functors for which our work in $\S 2$ is applicable.

All groups considered are finite. Any unusual notation will be explained as it is introduced.

We use $F(G)$ for the Fitting subgroup of the group $G$, and $F_{2}(G)$ is the subgroup of $G$ such that $F_{2}(G) / F(G)=F(G / F(G))$.

Finally recall that a Dedekind group is one in which each subgroup is normal (cf. [9, p. 139]). There is more than the obvious connection between Dedekind groups and those groups in which normality is a transitive relation (see $[\mathbf{9}, \S 13.4]$ ).

2. Subgroups which satisfy Frattini arguments. The Frattini argument is a familiar one. In various contexts it is used to factorize groups. Here we isolate a few of these contexts with the definitions below and study the resulting concepts.

2.1 Definition. Let $U \leq G$.

Received by the editors July 21, 1986. Portions of this paper were presented in August 1985 to the Groups-St. Andrews Conference by the first author.

1980 Mathematics Subject Classification (1985 Revision). Primary 20D99; Secondary 20D10, $20 \mathrm{D} 30$.

Key words and phrases. Subgroup functor, $t$-groups, Frattini-argument. 
(i) $U$ satisfies the weak Frattini argument in $G$ provided for each $K \triangleleft G$ with $U \leq K, G=K N_{G}(U)$,

(ii) $U$ satisfies the Frattini argument in $G$ provided for each $K \triangleleft G, G=$ $K N_{G}(U \cap K)$, and

(iii) $U$ satisfies the strong Frattini argument in $G$ provided for every $H \leq G$ with $U \leq H, U$ satisfies the Frattini argument in $H$.

As the terminology is meant to convey, the strong Frattini argument implies the Frattini argument. Likewise, the Frattini argument implies the weak Frattini argument. The successively stronger definitions are the result of adding a persistence condition. For instance, $U$ satisfies the Frattini argument in $G$ exactly when $U \cap K$ satisfies the weak Frattini argument in $G$ for each $K \triangleleft G$.

The notion that a subgroup satisfies the Frattini argument was incisive to the development in $\S 7$ of [2]. Obviously, normal subgroups and maximal subgroups satisfy the strong Frattini argument.

2.2 PROPOSITION. If $U \triangleleft \triangleleft G$ and $U$ satisfies the weak Frattini argument in $G$, then $U \triangleleft G$.

Proof. Let $K \triangleleft G$ with $U \leq K$. That $G=K N_{G}(U)$ implies $U^{G}=U^{K}$. (Here we use the notation $A^{B}=\left\langle A^{b} \mid b \in B\right\rangle$ for any $A$ and $B$ subgroups of $G$.) Since $U \triangleleft \triangleleft G$, if $U \neq U^{G}$, there would be $W \triangleleft U^{G}=U^{U^{G}}$ such that $U \leq W<U^{G}$. This impossibility forces $U=U^{G}$.

This result reminds one of an analogous result about pronormality. We recall for $U \leq G, U$ is pronormal in $G$ provided for each $g \in G$ there is $x \in\left\langle U, U^{g}\right\rangle$ such that $U^{g}=U^{x}$. Many newer texts have sections and problems dealing with pronormality. We will assume this is a familiar topic.

A related notion is "normally embedded." $U$ is normally embedded in $G$ provided $U \leq G$ such that for each prime $p$, there is a normal subgroup $N_{p}$ for which a Sylow $p$-subgroup of $U$ is a Sylow $p$-subgroup of $N_{p}$. The paper by Chambers [4] provides much information about subgroups with this property. In particular, if $G$ is solvable, any normally embedded subgroup is pronormal.

In light of 2.2 we provide an example to distinguish these properties.

2.3 EXAMPLE. Let $G=S_{4}$, the symmetric group of degree 4 . Let $H=$ $\langle(1234)\rangle . H$ is pronormal in $G$, since any two distinct conjugates of $H$ generate $G$. $H$ does not satisfy the Frattini argument in $G$, for otherwise (letting $V_{4}$ be the normal subgroup $G$ of order 4) $H \cap V_{4}$ would be a normal subgroup of order 2 by 2.2 .

On the other hand, if $U=\langle(12)\rangle, U$ satisfies the Frattini argument in $G$ since $U$ intersects each proper normal subgroup trivially. $U$ is not pronormal in $G$, for if it were, $U$ would be both pronormal and subnormal, and hence normal in a Sylow 2-subgroup of $G$ containing $U$. This is not the case.

2.4 Proposition. Let $U \leq G$.

(i) If $U$ is pronormal in $G$, then $U$ satisfies the weak Frattini argument in $G$.

(ii) If $N \triangleleft G$ and $U$ satisfies the Frattini argument in $G$, then $U N / N$ satisfies the Frattini argument in $G / N$.

(iii) If $N \triangleleft G$ and $U$ satisfies the weak Frattini argument in $G$, then $U N / N$ satisfies the weak Frattini argument in $G / N$. 
(iv) If $U$ satisfies the strong Frattini argument in $G$ and $G$ is solvable, then $U$ is pronormal in $G$.

Proof. (i) Let $K \triangleleft G$ with $U \leq K$. Then if $g \in G,\left\langle U, U^{g}\right\rangle \leq K$. But $U$ is pronormal and so there is $k \in K$ such that $U^{k}=U^{g}$. Hence $G=K \cdot N_{G}(U)$.

(ii) Suppose $N \triangleleft G$ and $U$ satisfies the Frattini argument in $G$. For $K \triangleleft G$ with $N \leq K, G=K \cdot N_{G}(U \cap K) \leq K \cdot N_{G}(U N \cap K)$ since $U N \cap K=(U \cap K) N$. Consequently $U N / N$ satisfies the Frattini argument in $G / N$.

(iii) Same proof as for (ii) works with restricted $K \triangleleft G$.

(iv) Here we use the fact that if for any $N \triangleleft G, U N$ is pronormal in $G$ and $U$ is pronormal in $N_{G}(U N)$, then $U$ is pronormal in $G$.

Proceed using induction on $|G|$ and let $N$ be a minimal normal subgroup of $G$. From (iii), it is apparent that $U N / N$ is pronormal in $G / N$. From this it follows easily that $U N$ is pronormal in $G$.

If $N_{G}(U N)<G$, then since $U$ satisfies the strong Frattini argument in $N_{G}(U N)$, $U$ would be pronormal in $N_{G}(U N)$.

It remains to consider the case $U N \triangleleft G$. By the Frattini argument, $G=$ $U N N_{G}(U)=N \cdot N_{G}(U)$. So for any $g \in G$, there is $n \in N$ such that $U^{n}=U^{g} \leq$ $U N$. If $U N<G$, then by induction $U$ is pronormal in $U N$ and so $U$ is pronormal in $G$.

Finally, if $G=U N$, the solvability of $G$ forces $U$ to be pronormal in $G$ since then either $U=G$ or $U$ is a maximal subgroup of $G$.

Our intent is to be more encyclopedic about these properties and their interrelations in subsequent work. Here we produce only what we intend to use in $\S 3$.

Recall that a metanilpotent group $G$ is one in which $G=F_{2}(G)$. We denote the class of metanilpotent groups by $\mathcal{N}^{2}$. If $G \in \mathcal{N}^{2}$, then $G$ is solvable and all quotients and subgroups of $G$ are metanilpotent.

2.5 Proposition. Let $G \in \mathcal{N}^{2}, p$ a prime and $U$ a $p$-subgroup of $G$. The following are equivalent:

(i) $U$ satisfies the strong Frattini argument in $G$,

(ii) $U$ satisfies the weak Frattini argument in $G$,

(iii) $U$ is normally embedded in $G$,

(iv) $U$ is pronormal in $G$.

Proof. That (i) implies (ii) is clear. To deduce that (ii) implies (iii), use induction on $|G|$.

Let $N$ be a minimal normal subgroup of $G$. From 2.4(ii) and induction, there is $K \triangleleft G$ such that $U N / N \in \operatorname{Syl}_{p}(K / N)$. If $N$ is a $p^{\prime}$-group, then $U \in \operatorname{Syl}_{p}(K)$ and then $U$ would be normally embedded in $G$.

On the other hand, if each minimal normal subgroup of $G$ is a $p$-group, then $F(G)$ is a $p$-group and consequently $F(G)=\operatorname{Syl}_{p}(G)$. Hence $U \leq F(G)$. But then $U$ is subnormal in $G$ and then by $2.2 U \triangleleft G$.

That (iii) implies (iv) is valid in any solvable group as is shown by Chambers [4].

Finally to prove that (iv) implies (i), we again use induction on $|G|$. Observing that if $U \leq S \leq G$ then $U$ is pronormal in $S$, it is only necessary to show that $U$ satisfies the Frattini argument in $G$. 
Let $K \triangleleft G$. Suppose then $N$ is a minimal normal subgroup of $G$. It is easy to see that $U N / N$ is pronormal in $G / N$ and so by induction and considering inverse images, $G=K \cdot N_{G}(U N \cap K N)$.

If $O_{p^{\prime}}(G)=1$, then $F(G)=\operatorname{Syl}_{p}(G)$ and so $U \leq F(G)$. But then $U$ is both pronormal and subnormal in $G$ and so $U \triangleleft G$.

We may then suppose $N$ is a $p^{\prime}$-group. Note that then $U N \cap K N=(U \cap K) N$ and so $N_{G}(U N \cap K N)=N_{G}((U \cap K) N)=N N_{G}(U \cap K)$ by a standard Frattini argument.

If $N \leq K$, then from above $G=K \cdot N_{G}(U \cap K)$. On the other hand, if $N \cap K=1, N \leq C_{G}(K) \leq N_{G}(U \cap K)$. Thus, from above, we see that in all cases, $G=K N_{G}(U \cap K)$ and so $U$ satisfies the Frattini argument in $G$.

3. Inclusion classes and $t$-groups. We now return to investigate functors and Fitting functors as defined in the introduction.

Given two functors $f$ and $g$, we define $f \subseteq g$ provided $f(G) \subseteq g(G)$ for each group $G$. This inclusion relation defines a partial ordering on a collection of functors.

For some useful functors, let us establish notation for this section. For a group $G$,

$s(G)=\{U \mid U \leq G\}$,

$\mathrm{w}(G)=\{U \mid U \leq G, U$ satisfies the weak Frattini argument in $G\}$,

$a(G)=\{U \mid U \leq G, U$ satisfies the Frattini argument in $G\}$,

$\$(G)=\{U \mid U \leq G, U$ satisfies the strong Frattini argument in $G\}$.

From $\S 2$, we have $\$ \subseteq a \subseteq \mathrm{w} \subseteq s$.

The inclusion relation can be used to obtain some isomorphy classes of groups.

3.1 Definition. Given two functors $f, g$, the inclusion class of $f$ in $g$ is $I(f, g)=\{G \mid f(G) \subseteq g(G)\}$.

The first result is trivial but of such usefulness we make special mention of it.

3.2 REMARK. Let $f, g, h$ be functors with $g \subseteq h$. Then

(i) $I(f, g) \subseteq I(f, h)$,

(ii) $\Sigma(h, f) \subseteq I(g, f)$.

The origin of this paper traces back to the question: "What can be said of a group in which each subgroup satisfies the Frattini argument?" In seeking an answer, we were led back to work of Gaschütz [5]. Recall that a group $G$ is called a $T$-group if normality is a transitive relationship in $G$. A solvable $T$-group is called a $t$-group. These $t$-groups are well understood (cf. Robinson $[\mathbf{9}, \S 13.4]$ ).

3.3 THEOREM (GASCHÜTZ [5]). Let $G$ be a solvable group. Then $G$ is a $t$ group if and only if there is $L \triangleleft G$ such that (1) $L$ is a Hall subgroup of $G,(2)$ $G / L$ is a Dedekind group, and (3) each subgroup of $L$ is normal in $G$.

3.4 THEOREM. Let $G$ be a solvable group. Then the following are equivalent:

(i) $G$ is a t-group.

(ii) $G \in I(s, \$)$.

(iii) $G \in I(s, \mathrm{w})$.

PROOF. (i) $\Rightarrow$ (ii). One consequence of 3.3 is that every subgroup of a $t$-group is a $t$-group. Thus it is sufficient to show if $G$ is a $t$-group, then $G \in I(s, a)$.

Let $U \subseteq G$ and $K \triangleleft G$ with $G$ a $t$-group. Let $L \triangleleft G$ satisfying (1), (2), and (3) of 3.3 . 
By (3), $U \cap K \cap L \triangleleft G$. Routinely, $U \cap K \cap L$ is a Hall subgroup of $U \cap K$. By (2) of 3.3, $(U \cap K) L \triangleleft G$ and so $(U \cap K) L \cap K=(U \cap K)(L \cap K) \triangleleft G$. Consequently $U \cap K / U \cap K \cap L$ is a Hall subgroup of $(U \cap K)(L \cap K) / U \cap K \cap L$. From applying a standard Frattini argument in $G / U \cap K \cap L$ and returning to $G$, it follows $G=K N_{G}(U \cap K)$. Therefore $G \in I(s, \$)$.

That (ii) $\Rightarrow$ (iii) is clear.

(iii) $\Rightarrow$ (i). If $G \in I(s, \mathrm{w})$ and $H \triangleleft K \triangleleft G$, then $K \leq N_{G}(H)$ and $G=$ $K N_{G}(H)$. Thus $H \triangleleft G$ and so $G$ is a $t$-group.

If $G$ is a simple group, then each subgroup of $G$ satisfies the Frattini argument in $G$. So until otherwise noted: All groups are solvable.

We address the question: "For which functors $f$ does $I(f, a)=t$-groups?" From 3.4 and $3.2, t$-groups comprise the smallest class which can occur as $I(f, a)$ for any $f$. Many different functors may give the same inclusion class. Thus it seems reasonable to seek $f$ such that $I(f, a)=I(s, a)$, but $f$ is small in the inclusion partial ordering. Since the Frattini argument is defined to be persistent in normal subgroups, we consider, mainly, Fitting functors.

Let us list some Fitting functors which appear to be valid candidates. For crossreference and motivation, we refer to the articles by Peng [6] and Robinson [7, 8].

3.5 Definition. For a group $G$, define

$l(G)=\{U \mid U$ is a cyclic $p$-group for some prime $p\}$,

$h(G)=\left\{U \mid U \in l(G), U \leq F_{2}(G)\right\}$,

$k(G)=\{U \mid U \in h(G), U \leq F(G)\}$.

Obviously $k \subseteq h \subseteq l$. From Beidleman, Brewster, and Hauck [2], it is easily deduced that $h, k$, and $l$ are Fitting functors. We can identify $I(k, a)$.

Let $\mathcal{G}=\{G \mid$ if $U \leq F(G)$, then $U \triangleleft G\}$.

3.6 Proposition. $I(k, \mathrm{w})=\mathcal{G}=I(k, a)$.

Proof. If $G \in I(k, \mathrm{w})$, then every cyclic $p$-subgroup of $F(G)$ satisfies the Frattini argument. Since $F(G)$ is a nilpotent subgroup of $G$, each cyclic $p$-subgroup of $F(G)$ is also subnormal. Hence, by 2.2 each cyclic $p$-subgroup of $F(G)$ is normal. Easily then, $G \in \mathcal{G}$.

Obviously $G \subseteq I(k, a) \subseteq I(k, \mathrm{w})$.

It is apparent that if $N \triangleleft G \in \mathcal{G}$, then $N \in \mathcal{G}$. However, the following example will show subgroups and quotients of groups in $\mathcal{G}$ need not be in $\mathcal{G}$, nor are groups in $\mathcal{G}$ necessarily $t$-groups.

3.7 EXAMPLE. Let $S_{3}$ denote the symmetric group of degree 3 and $H$ the nonabelian group of order 21. Let $G=S_{3} \times H$.

Certainly $G \in \mathcal{G}$. If $Q \in \operatorname{Syl}_{7}(G)$, then $G / Q \notin \mathcal{G}$. Similarly a Hall $\{2,3\}$ subgroup of $G$ does not belong to $\mathcal{G}$. Since subgroups of $t$-groups are $t$-groups, $G$ is not a $t$-group.

3.8 PROPOSITION. $I(h, a)=t$-groups $=I(h, \mathrm{w})$.

Proof. By 3.4 and 3.2, if $G$ is a $t$-group, $G \in I(h, a) \subseteq I(h, \mathrm{w})$.

On the other hand, if $G \in I(h, \mathrm{w})$, then $G \in I(k, \mathrm{w}) \subset \bar{N}^{2}$. Hence, $h(G)=l(G)$ and so by $2.5, G \in I(l, a)$. Thus by 2.5 and remarks in Robinson [8, p. 778], $G$ is a $t$-group. 
To reach a smaller functor is possible. Let $h_{0}(G)$ be the maximal members of $h(G) . h_{0}$ is not a Fitting functor, but we get the following corollaries, since each subgroup of a cyclic group is characteristic.

3.9 COROLlaRY. I $\left(h_{0}, a\right)=t$-groups.

3.10 COROLLARY. If $f$ is any functor such that $h_{0} \subseteq f$, then $I(f, a)=t$ groups.

3.11 ExAMPLE. Let $g(G)=\{U \mid U$ is a cyclic $p$-subgroup of $G\}$ and let $g_{0}(G)$ be the maximal elements in $g(G)$. From 3.10, and the argument showing 3.9, $I\left(g_{0}, a\right)=t$-groups.

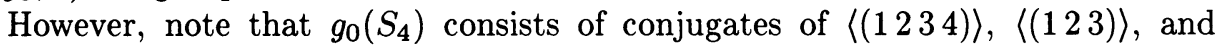
$\langle(12)\rangle$, each of which satisfies the weak Frattini argument in $S_{4}$. Thus $I\left(g_{0}, \mathrm{w}\right) \neq$ $I\left(g_{0}, a\right)$.

Finally we comment on the solvability hypotheses that have been inherent since 3.3. Recall a $p$-nilpotent group is one with a normal $p$-complement.

3.12 LEMMA. If $p$ is the smallest prime divisor of $|G|$ and each cyclic $p$ subgroup of $G$ satisfies the strong Frattini argument in $G$, then $G$ is p-nilpotent.

ProOF. Let $U$ be a $p$-subgroup of $G$. If $V$ is a cyclic group with $V \leq U$, then $V$ satisfies the Frattini argument in $N_{G}(U)$. By 2.2, $V \triangleleft N_{G}(U)$ and so $N_{G}(U) \leq N_{G}(V)$. Also

$$
C_{G}(U)=\bigcap\left\{C_{G}(V) \mid V \text { is a cyclic subgroup of } U\right\} \text {. }
$$

But for each such cyclic $V, N_{G}(V) / C_{G}(V)$ is a $p$-group since $N_{G}(V) / C_{G}(V)$ is both a section of $G$ and isomorphic to a subgroup of $\operatorname{Aut}(V)$. Then $N_{G}(U) / C_{G}(U)$ imbeds into the direct product $\prod\left\{N_{G}(V) / C_{G}(V) \mid V\right.$ is cyclic, $\left.V \leq U\right\}$ and so is a $p$-group. Thus by Frobenius' Theorem $[\mathbf{9}, 10.3 .2] G$ is $p$-nilpotent.

3.13 COROLlARY. Let $f$ be a functor. Suppose for each group $G$ that if $p$ is the smallest prime divisor of $|G|$, then $f(G)$ contains all cyclic p-subgroups of $G$. Then $I(f, \$)$ consists entirely of solvable groups.

The proof is omitted since it is a straight induction from 3.12. Utilizing the solvability of groups of odd order, the following is equally obvious.

3.14 COROLLARY. Let $f$ be a functor such that $f(G)$ contains all cyclic 2subgroups of $G$. If $G \in I(f, \$)$, then $G$ is solvable.

\section{REFERENCES}

1. D. Barnes and O. H. Kegel, Gaschütz functors on finite soluble groups, Math. Z. 94 (1966), $132-142$.

2. J. C. Beidleman, R. Brewster, and P. Hauck, Fittingfunctoren in endlichen aufiösbaren Gruppen. I, Math. Z. 182 (1983), 359-384.

3. __ Fitting functors in finite solvable groups. II, Proc. Cambridge Philos. Soc. 101 (1987), 37-55.

4. G. A. Chambers, p-normally embedded subgroups of finite soluble groups, J. Algebra 16 (1970), 442-455.

5. W. Gaschütz, Gruppen in denen das Normalteilersein transitiv ist, J. Reine Angew. Math. 198 (1957), 87-92. 
6. T. A. Peng, Finite groups with pronormal subgroups, Proc. Amer. Math. Soc. 20 (1969), 232234.

7. D. J. S. Robinson, A note on finite groups in which normality is transitive, Proc. Amer. Math. Soc. 19 (1968), 933-938.

8. __ Groups which are minimal with respect to normality being intransitive, Pacific J. Math. 31 (1969), 777-785.

9. _ A course in the theory of groups, Graduate Texts in Math., Vol. 80, Springer-Verlag, Berlin and New York, 1982.

10. W. Sudbrock, Sylowfunctionen in endlichen Gruppen, Rend. Math. Sem. Univ. Padova 36 (1966), 158-184.

Department of Mathematical Sciences, SUNY at Binghamton, Binghamton, NEW YORK 13901

Department of Mathematics, Ohio State University, Columbus, Ohio 43210 